\title{
A Fine-Grained Approach for Automated Conversion of JUnit Assertions to English
}

\author{
Danielle Gonzalez \\ Rochester Institute of Technology \\ Rochester, NY, USA \\ dng2551@rit.edu
}

\author{
Suzanne Prentice \\ University of South Carolina \\ Columbia, SC, USA \\ suzannep@sc.edu
}

\author{
Mehdi Mirakhorli \\ Rochester Institute of Technology \\ Rochester, NY, USA \\ mxmvse@rit.edu
}

\begin{abstract}
Converting source or unit test code to English has been shown to improve the maintainability, understandability, and analysis of software and tests. Code summarizers identify 'important' statements in the source/tests and convert them to easily understood English sentences using static analysis and NLP techniques. However, current test summarization approaches handle only a subset of the variation and customization allowed in the JUnit assert API (a critical component of test cases) which may affect the accuracy of conversions. In this paper, we present our work towards improving JUnit test summarization with a detailed process for converting a total of 45 unique JUnit assertions to English, including 37 previously-unhandled variations of the assertThat method. This process has also been implemented and released as the AssertConvert tool. Initial evaluations have shown that this tool generates English conversions that accurately represent a wide variety of assertion statements which could be used for code summarization or other NLP analyses.
\end{abstract}

\section{CCS CONCEPTS}

- Software and its engineering $\rightarrow$ Software testing and debugging;

\section{KEYWORDS}

Software Testing, JUnit, Code Summarization, NLP

\section{ACM Reference Format:}

Danielle Gonzalez, Suzanne Prentice, and Mehdi Mirakhorli. 2018. A FineGrained Approach for Automated Conversion of JUnit Assertions to English. In Proceedings of the 4th ACM SIGSOFT International Workshop on NLP for Software Engineering (NL4SE '18), November 4, 2018, Lake Buena Vista, FL, USA. ACM, New York, NY, USA, 4 pages. https://doi.org/10.1145/3283812. 3283819

\section{INTRODUCTION}

Code is a volatile artifact that must be written, maintained, and understood by many people with different experience and expertise. This has motivated the creation of automated code summarization tools, which analyze code, extract 'important' statements, and

Permission to make digital or hard copies of all or part of this work for personal or classroom use is granted without fee provided that copies are not made or distributed for profit or commercial advantage and that copies bear this notice and the full citation on the first page. Copyrights for components of this work owned by others than ACM must be honored. Abstracting with credit is permitted. To copy otherwise, or republish, to post on servers or to redistribute to lists, requires prior specific permission and/or a fee. Request permissions from permissions@acm.org.

NL4SE '18, November 4, 2018, Lake Buena Vista, FL, USA

(C) 2018 Association for Computing Machinery.

ACM ISBN 978-1-4503-6055-5/18/11 .\$15.00

https://doi.org/10.1145/3283812.3283819 produce natural language summaries. Through these summaries it is easier to gain a quick understanding of what the code is actually doing. This benefits maintenance, verification, and traceability activities. Many existing works have shown the feasibility and benefits of this approach by summarizing Java methods and classes $[3,7,8,10,11]$. Recently, these works have been adapted for the summarization of unit test code, and summaries of JUnit test cases have been shown to improve the understandability of test cases $[4,5,9,12]$. While these test summarization approaches result in readable summaries, they provide heuristics for converting only a limited subset of the complex-but-critical assert statement API. As a result, the applicability of these approaches for real large-scale projects is limited. In a JUnit test case, assert statements perform the 'core action' of the test by checking a condition after some manipulation of the code-under-test. The assert API provided by JUnit contains more than 45 unique combinations of conditions and parameters (expected/actual values, messages, deltas, etc.). Additional complexity arises from parameter order not being strictly enforced. Furthermore, the popular assertThat method gets almost no attention in previous work, despite being one of the most complex assertions provided by JUnit (there are over 40 unique variations of this method outlined in the API). Due to the critical role assert statements play in a JUnit test case, it is important that all possible variations are considered and properly converted to create an accurate summarization approach applicable across a large number of software projects.

This paper has two contributions: first, we present a detailed approach for automatically converting 45 unique variations of JUnit assert statements from Java to English. Our approach replicates previous test summarization techniques that already cover 8 variations of assert method. However, we augment this existing body of work by presenting novel heuristics for converting 37 assertThat variations. In addition, the entire conversion process has been implemented and released as the AssertConvert tool ${ }^{1}$. A preliminary evaluation shows that developers find the English conversions to be accurate representations of many varieties of assert statements.

\section{METHODOLOGY}

To produce accurate English conversions of assert methods, it is important to consider 3 key characteristics of the assert API ${ }^{2}$ :

(1) The 'core' of an assert is its condition, represented as 9 unique methods (ex: assertEquals, assertNull, \& assertTrue). However some methods are overwritten multiple times to allow for different combinations of optional parameters, resulting in 36 unique combinations of assert conditions and parameters.

\footnotetext{
AssertConvert Tool: goo.gl/f9Z4xr

${ }^{2}$ http://junit.sourceforge.net/javadoc/org/junit/Assert.html
} 
Example: all of the following are valid: assertEquals (num1, num2), assertEquals("num1 not equal to num2", num1, num2), assertEquals (num1, num2,0.01), and assertEquals ("num1 not equal to num2", num1, num2,0.01)

(2) Developers do not always follow the API-suggested parameter order, so extra steps must be taken to verify which parameter is which.

(3) The assertThat method (not handled by existing approaches) accepts as parameters special methods known as 'matchers' that provide more detailed conditions. Special heuristics are needed to handle these extra levels of complexity and accurately convert assertThat methods to English. This is demonstrated in the example below. Parameter 1 is the actual value, and the allof matcher contains 2 nested matchers. It checks that all of the nested matchers' conditions are met by the actual value. Example: assertThat("myValue", allof(startsWith("my"), containsString("Val"))).

Taking these into consideration, we use a 3-step conversion process. (1) Identify the assert's parameters, (2) convert the expected and actual value parameters to English phrases, and (3) combine these phrases into a single English sentence based on the assert's condition. In the following subsections the heuristics our approach uses for this process are detailed. The special case of assert that is discussed separately from the other types of assertion in Section 2.3.

\subsection{Parameter Identification}

JUnit asserts can have up to 4 parameters, three of which are optional or not relevant in all methods. The one required parameter is known as the actual value, and is the result of performing some operation on the code-under-test. The message (displayed on assertion failure) parameter is optional but if present, it must be the first parameter. For methods such as assertNull the expected value is not needed. Otherwise, it acts as a supplement to the condition such as for assertEquals. Finally, there is an optional delta parameter which is accepted when two numeric values are being compared to allow for an acceptance 'margin'. Our approach to identifying parameters uses two sets of heuristics:

Part 1: Identifying Parameters by Number and Order The first step is to determine if the method contains optional parameters. Each heuristic is checked in the order listed below.

1 Parameter: This is the easiest case, and the lone parameter is marked as the actual value.

2 Parameters:

(1) If the first parameter is a string AND the condition is Null, Not Null, True, or False, then the first parameter is the assertion message and the second parameter is the actual value.

(2) Otherwise, the two parameters are the expected \& actual values. 3 Parameters:

(1) If the first parameter is a string AND the condition is Equals, Not Equals, Same, Not Same, or ArrayEquals, then the first parameter is the message.

(2) If the first parameter is a string AND the condition is That, then the first parameter is the message.

4 Parameters: In this case, we reason that the first and fourth parameters are the message and delta.

$\underline{\text { Part 2: Distinguishing Between Values Using Type-Based Heuristics }}$
For all assertions with $\geq 2$ parameters (except assertThat, see Section 2.3), the actual and expected values are identified using type-based heuristics adopted from Zhang et. al [12]. Any message parameters are discarded.

(1) If one parameter is a constant and the other is a method call, the constant is the expected value and the method call is the actual value.

Example: assertEquals(myObj.getId(), 2456) Although the intended order is assertEquals (expected, actual), the developer has switched the order, but this heuristic is still able to identify myObj.get $\operatorname{Id}()$ as the actual value.

(2) If both parameters are method calls, a check [12] is performed to see if one was invoked by the class under test. If so, this call is the actual value and the other call is the expected value. Example: assertEquals (aNum. toString(), obj.getID()) The first call is a call invoked by a generic type, and the second is invoked by an object from the code-under-test. The first parameter is the expected value and the second is the actual value.

(3) In all other cases, it is decided that the developer followed the intended order, so the first parameter is the expected value and the second parameter is the actual value.

\subsection{Condition-Based Templates for Generating Full English Sentences}

In order to properly construct English sentences, we have adopted a condition-based template approach. The condition is easily extracted by camel-case splitting the assertion method name and removing the term 'assert'. These rules are also adapted from previous work [ 9 , 12], and our novel approach for converting assert That statements is described in Section 2.3.

(1) assertTrue, assertFalse, assertNull, assertNotNull Disregarding a message if present, there is only the actual value parameter, and the camel-case split conditions are easily appended to the end of the actual value phrase.

Template: actual value phrase + " is " + condition

Example: assertNotNull (myNum) = "my num is not null".

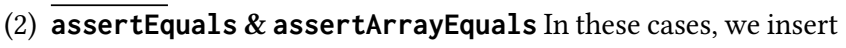
connecting terms between the two objects being compared and append the condition.

Template: actual value phrase + " and" + expected value phrase + "are equal."

Example: assertEquals (24, aNum)="a num and 24 are equal".

(3) assertSame \& assertNotSame These cases follow the same template as assertEquals \& assertArrayEquals but with small adjustments based for clarity.

Template: actual value phrase + "[is]/[is not $]$ identical to" + expected value phrase.

Example: assertNotSame (24, myNum) = "my num is not identical to $24 "$.

\subsection{The Special Case of assertThat}

assertThat is a special assertion that provides more fine-grain control over the condition being verified, even allowing multiple conditions to be checked at once. These methods can have up to 


\begin{tabular}{|c|c|c|c|}
\hline Matcher & Template & Example & Converted Example \\
\hline allof & matcher phrase + " and " + matcher phrase + " and " ... & assertThat("myValue", allOf(startsWith("my"), containsString("Val"))) & "starts with my and contains "Val"'"' \\
\hline any & "is type " + value phrase & assertThat(myCanoe, any(Canoe.class)); & "my Canoe is type Canoe" \\
\hline anyOf & matcher phrase + " or " + matcher phrase + " or " +. & assertThat("myValue", anyOf(startsWith("foo"), containsString("Val"))) & "starts with foo or contains "Val"'" \\
\hline anything & "is anything" & assetThat(myObj, anything()) & "my obj is anything" \\
\hline containsString & \begin{tabular}{|l|} 
"contains " + "value parameter" \\
\end{tabular} & assertThat("myString", contains("String")) & "''my string" contains "string"'"' \\
\hline endsWith & "ends with " + value phrase & assertThat("myString", endsWith("String")) & "my string ends with string" \\
\hline equalTo & "equals " + value phrase & assertThat("foo", equalTo("foo")) & "foo equals foo" \\
\hline everyltem & "every item in " + actual phrase + matcher phrase & assertThat(myArray, everyltem(startsWith("ba"))) & "every item in my array starts with ba" \\
\hline has Item (matchers) & " has an item that " + matcher phrase & assertThat(myArray, hasltem(endsWith("z"))) & "my array has an item that ends with z" \\
\hline hasltem(objects) & " has" + value phrase & assertThat(myArray, hasltem("baz")) & "my array has baz" \\
\hline hasltems(matchers) & " has items that " + matcher phrase + " and " + matcher phrase & assertThat(myArray, hasltems(endsWith("z"), endsWith("o"))) & "my array has items that ends with $\mathrm{z}$ and ends with o" \\
\hline hasltems(objects) & " has "+ value phrase + " and " + value phrase & assertThat(myArray, hasltems("baz", "foo")) & "my array has baz and foo" \\
\hline instanceOf & value phrase + " is an instance of" & assertThat(new Canoe(), instanceOf(Paddlable.class)); & "new Canoe is an instance of Paddlable" \\
\hline is & "is " + parameter phrase & assertThat(cheese, is(smelly)) & "cheese is smelly" \\
\hline is $\mathrm{A}$ & "is type " + value phrase & assertThat(cheese, isA(Cheddar)) & "cheese is type Cheddar" \\
\hline not & is not" + parameter phrase & assertThat(cheese, not(smelly)) & "cheese is not smelly" \\
\hline notNullValue & is not null" & assertThat(cheese, notNullValue()) & "cheese is not null" \\
\hline nullValue & is null" & assertThat(cheese, is(nullValue()) & "cheese is null" \\
\hline samelnstance & "is the same instance as " + value phrase & assertThat(myObj, samelnstance(Object)) & "myObj is the same instance as Object" \\
\hline startsWith & " starts with " + value phrase & assertThat("myString", startsWith("my")) & "my string starts with my" \\
\hline thelnstance & "is instance " + value phrase & assertThat(cheese, thelnstance(cheeselnst)) & "cheese is instance cheeselnst" \\
\hline Other Matchers & $\begin{array}{l}\text { The name of the matcher is camel case split and reconstructed } \\
\text { into a phrase by adding spaces between terms }\end{array}$ & assertThat(cheese,meetsCustomCondition()) & "cheese meets custom condition" \\
\hline
\end{tabular}

Figure 1: Templates for assertThat Core Matchers

three parameters; an optional 'reason' (analogous to 'message'), a required actual value, and a required 'matcher' method. The addition of these matcher parameters add significant complexity to parsing approaches, as many matchers are designed to accept one or more other matchers as parameters. There are over 20 'CoreMatchers' available in the Hamcrest API, plus custom matchers can be created. To demonstrate that it is possible to convert complicated assertions such as this, we have developed templates for 19 of the Hamcrest CoreMatchers (automatically recognized by JUnit) based on the API. Many matcher methods are also overloaded, so in combination with the parameter identification and conversion processes from Sections 2.1, 2.2, and 2.4, this approach can convert 37 variations of the assertThat method to English. After the first round of parsing (see Section 2.1) finds the actual value, these methods move to a special parser that extracts the condition and expected value(s) from the matcher(s). First, the "top level" condition is extracted and stored (ex: allof). Then, any nested matchers are recursively parsed and converted to a matcher phrase. Non-matcher parameters are converted to phrases using replicated heuristics (2.4). Finally, all phrases are combined with the "top level" condition.

Example: everyItem(startWi th ("My")) becomes "every item starts with "my"".

Figure 1 shows all the templates used to convert the matchers. For each template, if a matcher accepts only a type or value parameter (String, Object, etc.), the term value phrase is used to indicate the English phrase for that parameter. If a method accepts only matcher(s) as parameters, the term matcher phrase is used to mean the English phrase for that matcher and its own parameters. If a matcher has been overloaded and can accept either an type/value or matcher(s), the term parameter phrase is used.

\subsection{Converting Java Parameters to English}

To convert non-matcher assertion parameters to English, we have adapted previous approaches [3-5, 7-12]. We did not have access to the SWUM tool used by these teams to deconstruct method calls so instead we used the CoreNLP Part-of-Speech tagger [6], JavaParser ${ }^{3}$ and SimpleNLG [1]. This adaptation suits our purposes by adding more details to our conversions. For example, consider the assertion in Figure 2. The second parameter is a method

\footnotetext{
${ }^{3}$ https://javaparser.org
}

call. We applied the techniques from these works to identify the type of the method caller and expand the method name. Thus, cause.getStatusCode() becomes "http operation failed exception status code". We also use the JavaParser's SymbolSolver to replace variable names with their type to aid in comprehension.

\section{EVALUATION \& RESULTS}

As a preliminary check that our work towards generating understandable English representations of JUnit assert statements is on the right track, an evaluation was performed by 4 professional developers with JUnit testing experience.

\subsection{Evaluation Setup}

The process detailed in Section 2 was implemented as a tool named AssertConvert. 300 Java projects extracted from Github using GHTorrent [2] were given as input to the tool. From the approximately 2000 summaries generated, 25 were randomly selected. The randomly selected cases were reviewed to ensure at least 1 of each condition was included, otherwise a new random sample was reselected. For each, the original statement was presented followed by

EXPECTED OUTCOME
assertEquals(401, cause.getStatusCode())
SUMMARY
http operation failed exception status code and 401 are equal
Please rate, from a scale of 1 to 5 , how well the English
summary represents the information from the JUnit Assertion. 1
is "very poor" and 5 is "perfect" *
Very Poor

Figure 2: Example from Evaluation

its English conversion and the participant was asked to 'rate' each conversion on a scale of 1 to 5 based on how well the conversion acted as an understandable and accurate representation of each assert statement as shown in Figure 2. The scale was explained as a score of 1 was 'very poor' and a 5 was 'perfect'. It was also explained to the participant that some information, particularly variable names, were added to the summaries in order to prevent confusion. The evaluation used is available online ${ }^{4}$.

$\overline{{ }^{4} \text { https://goo.gl/forms/XafW9jx2zyAVi33V2 }}$ 
The participants in this evaluation were 4 professional developers, each with 4 or more years of experience with Java, and at least 1 year of experience regularly using JUnit for testing.

\subsection{Evaluation Results}

Figure 3 shows the range of average ratings for the 25 conversion included in the evaluation. The lowest average was 2.26, and the highest was 5 . We also wanted to know the percentage of conversions included in the evaluation received a majority positive rating across the 4 participants. For a 5-point rating scale, we considered a rating of 4 or 5 to be 'positive' (3 is 'neutral', and anything lower is 'negative'). Therefore, majority positive means that a conversion received at least 3 'positive' ratings. Table 1 reports the percentage of conversions in the evaluation which received 0 to 4 positive ratings. This shows that $84 \%(21)$ of the conversions received a majority positive rating from the participants.

Table 1: Rating Frequency for Quality of Assert Conversions

\begin{tabular}{|l|l|}
\hline \# of Positive Ratings & \% of Conversions \\
\hline 0 & $4 \%$ \\
\hline 1 & $0 \%$ \\
\hline 2 & $12 \%$ \\
\hline 3 & $\mathbf{6 0 \%}$ \\
\hline $\mathbf{4}$ & $\mathbf{2 4 \%}$ \\
\hline
\end{tabular}

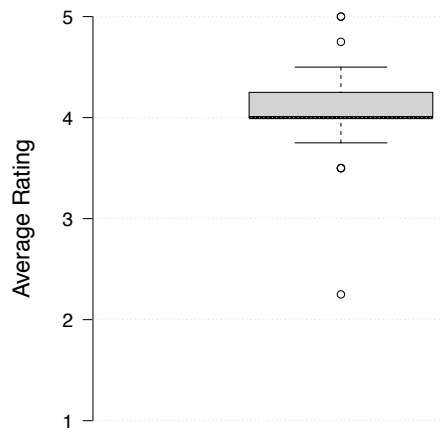

Figure 3: Average ratings for the 25 evaluated conversions

This indicates that the English conversions were, in most cases, understood by the developers and accurately represented the origin assert statement. With minor edits to the parameter conversion system, this value can be improved in future work.

\section{RELATED WORK}

Prior works produce English text that accurately conveys the purpose and actions of source/test code in an easily readable format. Moreno et al. have developed Java class-level summarization approaches [8] while others focus on the method level such as Sridhara et al. [11], McBurney et al. [7], Rastkar et al. [10] and Haiduc et al. [3]. There is less work translating Java test code all approaches (Zhang et al. [12], Kamimura and Murphy [4], Li et al. [5] and Panichella et al. [9] adapt the template-based source code approaches, and also use similar approaches for distinguishing parameters. However, in no case is such fine-grained attention paid to the conversion of the assert statements, especially the special cases for assertThat. Thus, this paper is complimentary to the previous works, with the hope of improving the accuracy of JUnit test summarization.

\section{CONCLUSIONS}

In this paper, we described our fine-grained approach for automatically generating English conversions for JUnit assertion statements. We replicated assertion conversion techniques and improved them by increasing the number of statement varieties that can be accurately converted. In total, 45 unique variations of assert statement are covered, including 37 variations of assert that that have not been handled prior. Preliminary evaluation shows the conversions can act as accurate representations of the assert statements. We feel that this comprehensive set of heuristics will increase the value and accuracy of English summaries of JUnit tests, enhancing test maintainability, and traceability. The process has also been implemented and released as the AssertConvert tool. Alone or as part of a full test case summary, these English conversions of assertions reveal valuable data about the test cases that can be easily analyzed at scale with other NLP techniques. In future work, we plan to make additional improvements to the parameter conversion heuristics, add heuristics for even more assert variations, and release the tool's source code so that the approach can be fully integrated into existing summarization tools.

\section{REFERENCES}

[1] Albert Gatt and Ehud Reiter. 2009. SimpleNLG: A realisation engine for practical applications. In Proceedings of the 12th European Workshop on Natural Language Generation. Association for Computational Linguistics, 90-93.

[2] Georgios Gousios. 2013. The GHTorrent dataset and tool suite. In Proceedings of the 10th Working Conference on Mining Software Repositories (MSR '13). IEEE Press, Piscataway, NJ, USA, 233-236. http://dl.acm.org/citation.cfm?id=2487085. 2487132

[3] Sonia Haiduc, Jairo Aponte, and Andrian Marcus. [n. d.]. Supporting program comprehension with source code summarization. In Proceedings of the 32nd ACM/IEEE International Conference on Software Engineering-Volume 2 (2010). ACM, 223-226. http://dl.acm.org/citation.cfm?id=1810335

[4] Manabu Kamimura and Gail C Murphy. 2013. Towards generating humanoriented summaries of unit test cases. In 2013 IEEE 21st International Conference on Program Comprehension (ICPC). IEEE, 215-218.

[5] Boyang Li, Christopher Vendome, Mario Linares-Vásquez, Denys Poshyvanyk, and Nicholas A Kraft. 2016. Automatically documenting unit test cases. In Software Testing, Verification and Validation (ICST), 2016 IEEE International Conference on. IEEE, 341-352.

[6] Christopher Manning, Mihai Surdeanu, John Bauer, Jenny Finkel, Steven Bethard, and David McClosky. 2014. The Stanford CoreNLP natural language processing toolkit. In Proceedings of 52nd annual meeting of the association for computational linguistics: system demonstrations. 55-60.

[7] Paul W. McBurney and Collin McMillan. [n. d.]. Automatic documentation generation via source code summarization of method context. In Proceedings of the 22nd International Conference on Program Comprehension (2014). ACM, 279-290. http://dl.acm.org/citation.cfm?id=2597149

[8] L. Moreno, J. Aponte, G. Sridhara, A. Marcus, L. Pollock, and K. Vijay-Shanker. [n. d.]. Automatic generation of natural language summaries for Java classes. In 2013 21st International Conference on Program Comprehension (ICPC) (2013-05). 23-32. https://doi.org/10.1109/ICPC.2013.6613830

[9] Sebastiano Panichella, Annibale Panichella, Moritz Beller, Andy Zaidman, and Harald C. Gall. 2016. The Impact of Test Case Summaries on Bug Fixing Performance: An Empirical Investigation. In Proceedings of the 38th International Conference on Software Engineering (ICSE '16). ACM, New York, NY, USA, 547-558. https://doi.org/10.1145/2884781.2884847

[10] S. Rastkar, G. C. Murphy, and A. W. J. Bradley. [n. d.]. Generating natural language summaries for crosscutting source code concerns. In 2011 27th IEEE International Conference on Software Maintenance (ICSM) (2011-09). 103-112. https://doi.org/10.1109/ICSM.2011.6080777

[11] Giriprasad Sridhara, Emily Hill, Divya Muppaneni, Lori Pollock, and K. VijayShanker. [n. d.]. Towards automatically generating summary comments for java methods. In Proceedings of the IEEE/ACM international conference on Automated software engineering (2010). ACM, 43-52. http://dl.acm.org/citation.cfm?id= 1859006

[12] Benwen Zhang, Emily Hill, and James Clause. [n. d.]. Towards automatically generating descriptive names for unit tests. In Automated Software Engineering (ASE), 2016 31st IEEE/ACM International Conference on (2016). IEEE, 625-636. http://ieeexplore.ieee.org/abstract/document/7582797/ 